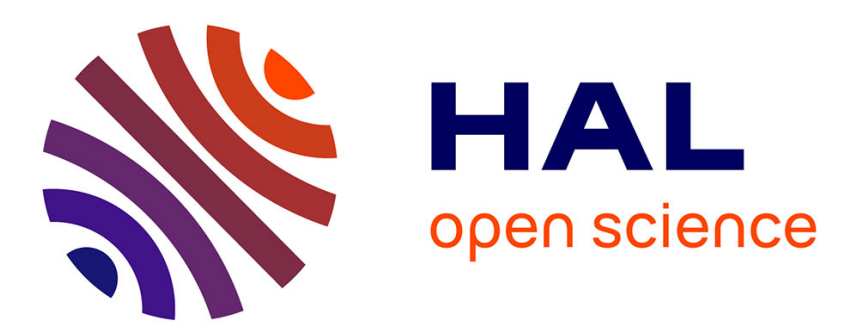

\title{
Reduced-order models for large-amplitude vibrations of shells including in-plane inertia
}

\author{
Cyril Touzé, M. Amabili, Olivier Thomas
}

\section{To cite this version:}

Cyril Touzé, M. Amabili, Olivier Thomas. Reduced-order models for large-amplitude vibrations of shells including in-plane inertia. Computer Methods in Applied Mechanics and Engineering, 2008, 197 (21-24), pp.2030-2045. 10.1016/j.cma.2008.01.002 . hal-00838878

\section{HAL Id: hal-00838878 \\ https://hal-ensta-paris.archives-ouvertes.fr/hal-00838878}

Submitted on 12 Mar 2016

HAL is a multi-disciplinary open access archive for the deposit and dissemination of scientific research documents, whether they are published or not. The documents may come from teaching and research institutions in France or abroad, or from public or private research centers.
L'archive ouverte pluridisciplinaire HAL, est destinée au dépôt et à la diffusion de documents scientifiques de niveau recherche, publiés ou non, émanant des établissements d'enseignement et de recherche français ou étrangers, des laboratoires publics ou privés. 


\title{
Reduced-order models for large-amplitude vibrations of shells including in-plane inertia
}

\author{
C. Touzé ${ }^{\mathrm{a}, *}$, M. Amabili ${ }^{\mathrm{b}}$, O. Thomas ${ }^{\mathrm{c}}$ \\ ${ }^{\text {a }}$ ENSTA-UME, Unité de recherche en Mécanique, Chemin de la Hunière, 91761 Palaiseau cedex, France \\ ${ }^{\mathrm{b}}$ Università di Parma, Parco Area delle scienze 181/A, Parma, Italy \\ ${ }^{\mathrm{c}}$ CNAM, Laboratoire de Mécanique des Structures et Systèmes Couplés, 2 rue Conté, 75003 Paris, France
}

\begin{abstract}
Non-linear normal modes (NNMs) are used in order to derive reduced-order models for large amplitude, geometrically non-linear vibrations of thin shells. The main objective of the paper is to compare the accuracy of different truncations, using linear and non-linear modes, in order to predict the response of shells structures subjected to harmonic excitation. For an exhaustive comparison, three different shell problems have been selected: (i) a doubly curved shallow shell, simply supported on a rectangular base; (ii) a circular cylindrical panel with simply supported, in-plane free edges; and (iii) a simply supported, closed circular cylindrical shell. In each case, the models are derived by using refined shell theories for expressing the strain-displacement relationship. As a consequence, in-plane inertia is retained in the formulation. Reduction to one or two NNMs shows perfect results for vibration amplitude lower or equal to the thickness of the shell in the three cases, and this limitation is extended to two times the thickness for two of the selected models.
\end{abstract}

Keywords: Shell vibrations; Non-linear normal modes; Model reduction; Geometrical non-linearity

\section{Introduction}

The large amplitude, geometrically non-linear vibrations of shells leads to complicated motions with typical non-linear phenomena. As a consequence, a large number of expansion functions is generally needed for discretizing the structure in order to obtain convergence through the Galerkin method. This results in large computational times that could be prohibitive in the context of simulation or control. For this reason, there is an important need for the definition of reduced-order models (ROMs), which capture the most salient features of the non-linear dynamics with a limited number of degrees-of-freedom (dofs).

Non-linear normal modes (NNMs) are defined in order to bypass the limitations of the linear normal modes (LNMs) in the non-linear range. The idea is to "decouple" as much as possible the motions in selected sub-spaces of the phase space. This is realized by imposing the invariance of sub-spaces as the key property that must be conserved when non-linearities come into play, and leads to the definition of NNMs as invariant manifolds of the phase space [1]. As the method used for computing the NNMs is purely non-linear, it is expected to give better results than using the LNMs, or modes obtained via the proper orthogonal decomposition (POD) method [2-5].

Several methods have then been proposed to compute the NNMs. They generally rely on an asymptotic development for approximating the invariant manifold [6-11]. More recently, purely numerical methods have been proposed [12-14], but they are often restricted either by complexity (i.e. computational time for calculating the ROMs) or by practicality, as once the complex geometry of the manifold is computed, one has to project the equations of motion onto it. In the context of shell vibrations, reduction with asymptotic NNMs has already been applied 
with success on a fluid-filled circular cylindrical shell described by the Donnells' shallow-shell assumptions [15], and compared with the POD method [2]. It also allows prediction of the type of non-linearity (hardening/softening behaviour) for shallow spherical shells [16].

In this study, the asymptotic NNM method is applied to shell structures, without the shallow-shell assumption. As a consequence, in-plane inertia is retained in the equations of motions, which are derived via a Lagrangian approach [1719]. In-plane inertia must be retained for shells that cannot be considered shallow. The effect of retaining or neglecting in-plane inertia has been addressed by Amabili [17] for closed circular cylindrical shells, and by Abe et al. [20] for curved panels but only in the linear (natural frequencies) part of their study. As a consequence of taking into account these additional degrees-of-freedom, the computation of the LNMs is tedious and is thus bypassed by using as expansion functions an ad hoc basis verifying the boundary conditions. As a consequence, linear coupling terms among the discretized oscillator equations are present.

The present study is focused on the computation of frequency-response curves for harmonically forced shells vibrating at large amplitude. As a complete bifurcation diagram (with stable and unstable solution branches) in the steady-state is sought, a continuation algorithm is naturally selected. In this context, structures discretized by finite-element based methods must obviously be reduced, as the number of degrees-of-freedom involved in a classical mesh is immediately too large to be treated by standard continuation algorithm. For this reason, the LNMs and NNMs are here used for producing this reduction.

The reduction method proposed in [15] is revised in order to take into account the linear coupling terms between oscillators. In particular, these terms link together flexural and in-plane motions. The procedure is automated and thus extends earlier results presented in [21] where the in-plane motions of a plate were slaved to the flexural motions by using an invariant manifold approach. Amongst other things, the developments presented herein on the reduction process with NNMs show that the method can easily handle any set of non-linear oscillators, as well as more refined shell theories. Consequently, the method has the potential for a large class of structural problems, and could be applied to finite elements analysis of shells, where the discretization functions, as well as the underlying shell theories are distinct from those used here [22], providing that an automated step computing the projection onto the linear modes have been programmed.

Three structures are selected in order to compare the results. A doubly curved panel, and a circular cylindrical panel, with the Donnell non-linear theory without the shallow-shell assumption, are first derived. Then a closed empty shell, the kinematics of which is expressed with the Flügge-Lur'e-Byrne non-linear theory, is studied. For each case, the reference solution is compared to ROMs with an increasing number of LNMs and NNMs. The amplitude of the external forcing is also varied in order to test the validity limits of the asymptotic approach used to generate the NNMs. As side result, upper validity limits, in terms of vibration amplitude with respect to the thickness of the shells, are derived for each model.

\section{Theoretical formulation}

\subsection{Selected cases}

Three cases have been selected for studying the reduction method provided by the asymptotic NNM formulation:

(i) A hyperbolic paraboloid panel with rectangular base, referred to as the HP panel in the following.

(ii) A circular cylindrical panel, referred to as the $\mathrm{CC}$ panel.

(iii) A closed circular cylindrical shell, referred to as the Flügge shell.

An exhaustive presentation of the HP panel is given in [18], whereas the CC panel and the Flügge shell are respectively documented in $[17,19]$. These shell models have been selected in order to draw out a complete picture of the reduction process in different situations. In particular, two different kinematics are used (Donnell's non-linear shell theory is used for case (i) and (ii), while case (iii) is governed by Flügge-Lur'e-Byrne non-linear theory), and the qualitative behaviour of the closed shell (case (iii)) is appreciably different from the two panels due to the symmetry of the problem.

The three shell models will be presented in a unified manner in this section, by emphasizing the general method used to obtain the equations of motions. As a consequence, only the common features of the three models are here described. Peculiar features, such as boundary conditions or projection functions used for discretization, will be reported in Sections 4-6.

Fig. 1 shows the geometry and coordinate system for a typical shell. This geometry can handle the three cases

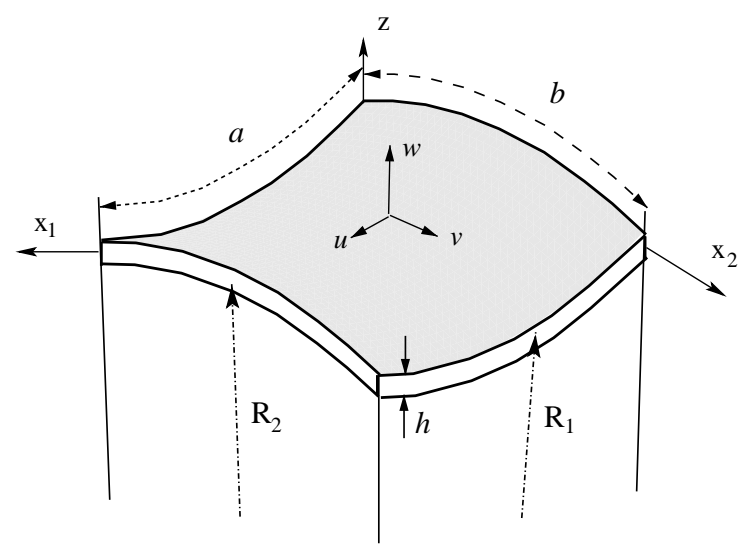

Fig. 1. Geometry and coordinate systems for the selected shells. 
and is thus used here as a generic model for presenting the equations of motions. The curvilinear coordinate system is denoted by $\left(O, x_{1}, x_{2}, z\right)$, with the origin $O$ at one edge of the panel. $R_{1}$ and $R_{2}$ (assumed to be independent of $x_{1}$ and $x_{2}$ ) are the principal radii of curvature, $a$ and $b$ are curvilinear length, and $h$ is the thickness. The membrane displacements are denoted by $u$ and $v$, and the normal displacement is $w$. In the following, $\mathscr{S}$ refers to the surface of the shell, i.e. $\mathscr{S}=[0, a] \times[0, b]$.

\subsection{Shell kinematics and external loads}

The shell kinematics is described by relating the strain components $\varepsilon_{1}, \varepsilon_{2}$ and $\gamma_{12}$ at an arbitrary point of the considered shell to the middle surface strains $\varepsilon_{1,0}, \varepsilon_{2,0}$ and $\gamma_{12,0}$, and to changes in the curvature and torsion of the middle surface $k_{1}, k_{2}$ and $k_{12}$ by

$\varepsilon_{1}=\varepsilon_{1,0}+z k_{1}$,

$\varepsilon_{2}=\varepsilon_{2,0}+z k_{2}$,

$\gamma_{12}=\gamma_{12,0}+z k_{12}$,

where $z$ is the distance of the arbitrary point from the middle surface.

For the HP and CC panels, Donnell's non-linear shell theory is used to express the strain-displacement relationship. The full expressions of the relationships between the middle surface strain-displacement and the changes in curvature and torsion can be found, for the HP panel in [18], and for the CC panel in [19]. For the Flügge shell, FlüggeLur'e-Byrne non-linear theory is used, leading to a more complicated relationship that is not reported here for the sake of brevity. The interested reader is referred to $[17,23,24]$ for complete expressions of the kinematics in that case.

The elastic strain energy $U_{S}$ is expressed with the classical assumption of plane stress. According to the geometry shown in Fig. 1, it reads

$$
\begin{aligned}
U_{S}= & \frac{1}{2} \int_{\mathscr{S}} \int_{-h / 2}^{h / 2}\left(\sigma_{1} \varepsilon_{1}+\sigma_{2} \varepsilon_{2}+\tau_{12} \gamma_{12}\right)\left(1+\frac{z}{R_{1}}\right) \\
& \times\left(1+\frac{z}{R_{2}}\right) \mathrm{d} S \mathrm{~d} z,
\end{aligned}
$$

where the stresses $\sigma_{1}, \sigma_{2}$ and $\tau_{12}$ are related to the strains for an homogeneous and isotropic material by

$$
\begin{aligned}
\sigma_{1} & =\frac{E}{1-v^{2}}\left(\varepsilon_{1}+v \varepsilon_{2}\right), \\
\sigma_{2} & =\frac{E}{1-v^{2}}\left(\varepsilon_{2}+v \varepsilon_{1}\right), \\
\tau_{12} & =\frac{E}{2(1+v)} \gamma_{12} .
\end{aligned}
$$

The kinetic energy $T_{S}$, by neglecting rotary inertia, reads

$T_{S}=\frac{1}{2} \rho h \int_{\mathscr{S}}\left(\dot{u}^{2}+\dot{v}^{2}+\dot{w}^{2}\right) \mathrm{d} S$, where $\rho$ is the mass density, and overdot is used for expressing time derivation.

The virtual work $W$ done by the external forces reads

$W=\int_{\mathscr{S}}\left(q_{x_{1}} u+q_{x_{2}} v+q_{z} w\right) \mathrm{d} S$

where $q_{x_{1}}, q_{x_{2}}$ and $q_{z}$ are the distributed forces per unit area acting in $x_{1}, x_{2}$ and $z$ directions respectively. For the three cases studied, a pointwise normal excitation due to the concentrated force $\tilde{f}$, with purely harmonic content, is considered:

$q_{x_{1}}=0, \quad q_{x_{2}}=0, \quad$ and

$q_{z}=\tilde{f} \delta\left(x_{1}-\tilde{x}_{1}\right) \delta\left(x_{2}-\tilde{x}_{2}\right) \cos \omega t$,

where $\left(\tilde{x}_{1}, \tilde{x}_{2}\right)$ is the position of the excitation point, and $\omega$ the excitation frequency. With this expression, Eq. (9) writes

$W(t)=\tilde{f} \cos (\omega t) w\left(x_{1}=\tilde{x_{1}}, x_{2}=\tilde{x_{2}}, t\right)$.

\subsection{Lagrange equations of motions}

The equations of motions are obtained via a Lagrange formulation. The displacements are expanded on a set of expansion functions $\phi_{m n}^{(u)}, \phi_{m n}^{(v)}, \phi_{m n}^{(w)}$, satisfying identically the boundary conditions

$$
\begin{aligned}
u\left(x_{1}, x_{2}, t\right) & =\sum_{m, n=1}^{M_{u}, N_{u}} u_{m n}(t) \phi_{m n}^{(u)}\left(x_{1}, x_{2}\right), \\
v\left(x_{1}, x_{2}, t\right) & =\sum_{m, n=1}^{M_{v}, N_{v}} v_{m n}(t) \phi_{m n}^{(v)}\left(x_{1}, x_{2}\right), \\
w\left(x_{1}, x_{2}, t\right) & =\sum_{m, n=1}^{M_{w}, N_{w}} w_{m n}(t) \phi_{m n}^{(w)}\left(x_{1}, x_{2}\right) .
\end{aligned}
$$

The explicit formulation for the selected expansion functions will be given in Section 4 for the HP panel, Section 5 for the CC panel, and Section 6 for the Flügge shell. The number of basis functions in each direction is free and governed by the integers $M_{u}, N_{u}, M_{v}, N_{v}, M_{w}$, and $N_{w}$. The damping forces are assumed to be of the viscous type. They are taken into account using Rayleigh's dissipation function

$F=\frac{1}{2} c \int_{\mathscr{S}}\left(\dot{u}^{2}+\dot{v}^{2}+\dot{w}^{2}\right) \mathrm{d} S$,

where $c$ is the damping coefficient. In the remainder, modal damping is assumed, such that $c$ will give birth to modal damping factors, that are different from one mode to another.

Let $\mathbf{q}$ be the vector of generalized coordinates, gathering together all the unknown functions of time introduced by the expansions given in Eqs. (12)

$$
\begin{aligned}
& \mathbf{q}=\left[u_{m, n}, v_{m, n}, w_{m, n}\right]^{\mathrm{T}}, \quad m=1, \ldots, M_{u}, M_{v}, M_{w}, \\
& n=1, \ldots, N_{u}, N_{v}, N_{w} .
\end{aligned}
$$


In the remainder, $P$ refers to the dimension of $\mathbf{q}$, i.e. the number of generalized coordinates used for discretizing the shell. The generic element of $\mathbf{q}$ is denoted by $q_{p}$.

The generalized forces $Q_{p}$ are obtained by differentiation of Rayleigh's dissipation function $F$, provided by Eq. (13), and of the virtual work $W$ done by external forces, Eq. (9). It reads

$Q_{p}=-\frac{\partial F}{\partial \dot{q}_{p}}+\frac{\partial W}{\partial q_{p}}$.

The Lagrange equations of motion can now be expressed:

$\forall p=1, \ldots, P: \quad \frac{\mathrm{d}}{\mathrm{d} t}\left(\frac{\partial T_{S}}{\partial \dot{q}_{p}}\right)-\frac{\partial T_{S}}{\partial q_{p}}+\frac{\partial U_{S}}{\partial q_{p}}=Q_{p}$.

As a consequence of the kinetic energy expression, it is found that $\partial T_{S} / \partial \dot{q}_{p}=0$. The derivation of the elastic strain energy $U_{S}$ with respect to $q_{p}$ shows very complicated expressions involving quadratic and cubic non-linear coupling terms among the equations. Finally, in the three considered cases, the result of the discretization gives a set of coupled non-linear oscillator equations to solve. They writes

$$
\begin{aligned}
\ddot{q}_{p} & +2 \zeta_{p} \omega_{p} \dot{q}_{p}+\sum_{i=1}^{P} z_{i}^{p} q_{i}+\sum_{i, j=1}^{P} z_{i, j}^{p} q_{i} q_{j}+\sum_{i, j, k=1}^{P} z_{i, j, k}^{p} q_{i} q_{j} q_{k} \\
& =f_{p} \cos (\omega t) .
\end{aligned}
$$

Modal damping in Eq. (17) is considered in the classical form $2 \zeta_{p} \omega_{p} \dot{q}_{p}$, and $\mathbf{f}=\left[f_{1} \ldots f_{P}\right]^{\mathrm{T}}$ is the vector of the projected external forcing considered.

\subsection{Discussion on in-plane inertia}

In-plane inertia must be retained for shells that cannot be considered shallow. Moreover, for higher vibration modes of shallow shells, in-plane inertia must also be retained. A specific case is a closed circular cylindrical shells for modes with a number of circumferential waves lower than four or five. However, even for shallow curved panels and closed shells with circumferential wavenumber equal or higher than five, in-plane inertia can play a significant role, as shown by Amabili [17]. For very shallow panels, the effect of in-plane inertia can be negligible on both natural frequencies and non-linear responses.

The drawback of retaining in-plane inertia is that additional degrees-of-freedom must be taken into the expansion as a consequence that the simplified Donnell's shallow-shell formulation cannot be used. Secondly, the computation of the eigenmodes can become more difficult. Analytical eigenmodes of panels with in-plane inertia are simply obtained in case of simply supported boundary conditions. In other cases, the formulation become much more complex and it is convenient to approach the problem numerically, e.g. by using the Rayleigh-Ritz method. For these reasons, ad hoc expansion functions are here used for discretizing the problem.

\section{Reduced-order modeling}

\subsection{Reference solution}

The response of the three selected shells to concentrated harmonic force is sought. The excitation frequency $\omega$ is selected close to the first eigenfrequency of the systems. Frequency-response curves are numerically obtained with the software AUTO [25], by continuation of the solutions with the pseudo-arclength method. Bifurcation analysis, branch switching and computation of the stability is performed by AUTO. In practice, the shell response to harmonic excitation is found in two steps. Firstly, the frequency $\omega$ is set apart of the eigenfrequency, and the excitation amplitude is used as bifurcation parameter. From the stable state at rest, the excitation amplitude is raised from zero to the desired magnitude. Once this value is reached, the excitation frequency is then selected as bifurcation parameter to obtain the frequency-response curve.

The reference solution is obtained with the described method, applied to Eq. (17). The convergence of the solutions with respect to the number $P$ of generalized coordinates retained has already been done in previous studies. It has been shown that, for the HP panel, $P=22$ basis functions were needed for obtaining convergence [18]. For the CC panel, 19 basis functions were needed [19], whereas convergence was obtained for the Flügge shell with 16 basis functions [17]. As a consequence of these large values, computation time associated with the numerical simulations with AUTO for obtaining a single frequency-response curve are large.

The next two subsections describe two strategies for reducing the dimension $P$, i.e. the number of oscillator equations to simulate to recover the correct behaviour. These reduction strategies are crucial in order to reduce the important computational time associated to simulations with a large number of dofs, that are unavoidable when dealing with geometrically non-linear structures.

Direct integration of the equations of motion is also performed in order to obtain time responses of the selected structures. The Gear's BDF method, implemented in the DIVPAG routine of the Fortran Library IMSL, has been selected to handle the high-dimensionality of the problem associated with stiff behaviour.

\subsection{Numerical computation of the linear normal modes}

The first idea for reducing the size of the system is to use the linear normal modes (LNMs). Let $\mathbf{L}=\left[z_{i}^{p}\right]_{p, i}$ be the linear part of Eq. (17), and $\mathbf{P}$ the matrix of eigenvectors (numerically computed) of $\mathbf{L}$ such that: $\mathbf{P}^{-1} \mathbf{L P}=\boldsymbol{\Lambda}$, with $\boldsymbol{\Lambda}=\operatorname{diag}\left[\omega_{p}^{2}\right]$, and $\omega_{p}$ the eigenfrequencies of the structure. A linear change of coordinates is computed, $\mathbf{q}=\mathbf{P X}$, where $\mathbf{X}=\left[X_{1} \ldots X_{P}\right]^{\mathrm{T}}$ is, by definition, the vector of modal coordinates. Application of $\mathbf{P}$ makes the linear part diagonal, so that the dynamics can now be expressed in the eigenmodes basis, and reads, $\forall p=1, \ldots, P$ : 


$$
\begin{aligned}
\ddot{X}_{p} & +2 \zeta_{p} \omega_{p} \dot{X}_{p}+\omega_{p}^{2} X_{p}+\sum_{i, j=1}^{P} g_{i j}^{p} X_{i} X_{j} \\
& +\sum_{i, j, k=1}^{P} h_{i j k}^{p} X_{i} X_{j} X_{k} \\
& =F_{p} \cos (\omega t) .
\end{aligned}
$$

The application of $\mathbf{P}$ let the viscous damping unchanged, and $\mathbf{F}=\mathbf{P}^{-1} \mathbf{f}=\left[F_{1} \ldots F_{P}\right]^{\mathrm{T}}$ is the new vector of modal forces. The quadratic and cubic non-linear coupling coefficients $\left\{g_{i j}^{p}\right\}$ and $\left\{h_{i j k}^{p}\right\}$ are computed from the $\left\{z_{i, j}^{p}\right\}$ and $\left\{z_{i, j, k}^{p}\right\}$ appearing in Eq. (17) with matrix operations involving $\mathbf{P}$. The dimension of $\mathbf{X}$ is $P$, but truncation can now be realized by keeping any number of LNMs. Let $P_{\mathrm{LNM}}$ be the dimension of the truncation operated in $\mathbf{X}$. Convergence studies will be realized by increasing $P_{\mathrm{LNM}}$ from 1 to $P$. Since the LNMs possesses some interesting properties (in particular orthogonality), it is awaited to obtain convergence for $P_{\mathrm{LNM}} \leqslant P$.

\subsection{Non-linear normal modes ( NNMs)}

Asymptotic approximation of the NNMs of the unforced structure is used to obtain further reduction of the size of the system. NNMs are defined as invariant manifold in phase space, tangent at the origin (representing the structure at rest) to the linear eigenmodes [1]. The procedure, based on normal form theory, is here briefly recalled. A complete description is provided in $[15,10]$.

A non-linear change of coordinates is performed, in order to express the dynamics within the phase space spanned by the invariant manifolds. The invariance property is the key that allows finding reduced-order models of lower dimension than those obtained using the eigenmodes [10]. The modal velocity $Y_{p}=\dot{X}_{p}$ is used to recast the dynamical equation (18) into its first-order form. The non-linear change of coordinates, up to the third order, is computed once and for all. It reads, $\forall p=1, \ldots, P$

$$
\begin{aligned}
X_{p}= & R_{p}+\sum_{i=1}^{P} \sum_{j \geqslant i}^{P}\left(a_{i j}^{p} R_{i} R_{j}+b_{i j}^{p} S_{i} S_{j}\right)+\sum_{i=1}^{P} \sum_{j=1}^{P} c_{i j}^{p} R_{i} S_{j} \\
& +\sum_{i=1}^{P} \sum_{j \geqslant i}^{P} \sum_{k \geqslant j}^{P}\left(r_{i j k}^{p} R_{i} R_{j} R_{k}+s_{i j k}^{p} S_{i} S_{j} S_{k}\right) \\
& +\sum_{i=1}^{P} \sum_{j=1}^{P} \sum_{k \geqslant j}^{P}\left(t_{i j k}^{p} S_{i} R_{j} R_{k}+u_{i j k}^{p} R_{i} S_{j} S_{k}\right), \\
Y_{p}= & S_{p}+\sum_{i=1}^{P} \sum_{j \geqslant i}^{P}\left(\alpha_{i j}^{p} R_{i} R_{j}+\beta_{i j}^{p} S_{i} S_{j}\right)+\sum_{i=1}^{P} \sum_{j=1}^{P} \gamma_{i j}^{p} R_{i} S_{j} \\
& +\sum_{i=1}^{P} \sum_{j \geqslant i}^{P} \sum_{k \geqslant j}^{P}\left(\lambda_{i j k}^{p} R_{i} R_{j} R_{k}+\mu_{i j k}^{p} S_{i} S_{j} S_{k}\right) \\
& +\sum_{i=1}^{P} \sum_{j=1}^{P} \sum_{k \geqslant j}^{P}\left(v_{i j k}^{p} S_{i} R_{j} R_{k}+\zeta_{i j k}^{p} R_{i} S_{j} S_{k}\right)
\end{aligned}
$$

The newly introduced variables, $\left(R_{p}, S_{p}\right)$, are respectively homogeneous to a displacement and a velocity, and are called the normal coordinates. The introduced coefficients in Eqs. (19) are the transformation coefficients, whose analytical expressions are given in [15]. Substitution of (19) into (18) gives the dynamics expressed in the invariantbased span of the phase space. It reads, $\forall p=1, \ldots, P$

$$
\begin{aligned}
& \ddot{R}_{p}+\omega_{p}^{2} R_{p}+2 \zeta_{p} \omega_{p} \dot{R}_{p}+\left(h_{p p p}^{p}+A_{p p p}^{p}\right) R_{p}^{3}+B_{p p p}^{p} R_{p} \dot{R}_{p}^{2} \\
& \quad+C_{p p p}^{p} R_{p}^{2} \dot{R}_{p}+R_{p}\left[\sum _ { j > p } ^ { N } \left[\left(h_{p j j}^{p}+A_{p j j}^{p}+A_{j p j}^{p}\right) R_{j}^{2}+B_{p j j}^{p} \dot{R}_{j}^{2}\right.\right. \\
& \left.+\left(C_{p j j}^{p}+C_{j p j}^{p}\right) R_{j} \dot{R}_{j}\right] \\
& \left.+\sum_{i<p}\left[\left(h_{i i p}^{p}+A_{i i p}^{p}+A_{p i i}^{p}\right) R_{i}^{2}+B_{p i i}^{p} \dot{R}_{i}^{2}+\left(C_{p i i}^{p}+C_{i p i}^{p}\right) R_{i} \dot{R}_{i}\right]\right] \\
& \quad+\dot{R}_{p}\left[\sum_{j>p}^{N}\left(B_{j p j}^{p} R_{j} \dot{R}_{j}+C_{j j p}^{p} R_{j}^{2}\right)+\sum_{i<p}\left(B_{i i p}^{p} R_{i} \dot{R}_{i}+C_{i i p}^{p} R_{i}^{2}\right)\right] \\
& \quad=F_{p} \cos (\omega t) .
\end{aligned}
$$

Eq. (20) is the normal form, up to the third order, of Eq. (18), computed without the forcing term on the right-hand side. The forcing term $\mathbf{F}=\left[F_{1} \ldots F_{P}\right]^{\mathrm{T}}$ is added after the non-linear change of coordinates on the normal oscillator equations. As a consequence of this treatment of the forcing term, the non-linear change of coordinate is time-independent. Hence two approximations have been used to build the reduced-order model based on NNMs. Firstly, the invariant manifolds are approximated via an asymptotic development up to order three. Secondly, time-independent NNMs are used to approximate time-dependent manifolds. In the mechanical context, a time-dependent formulation for computation of NNMs have been proposed by Jiang et al. [13,26], by adapting a numerical Galerkin procedure earlier developed by Pesheck et al. $[12,27]$. Their numerical results shows, amongst other things, that for moderate values of the forcing, the oscillations of the manifolds are small. Moreover, taking them into account requires a huge numerical effort.

Due to these two approximations, it is thus expected to obtain very good results for moderate values of the forcing and of the amplitude of the response. By increasing the amplitude of the forcing, the results are expected to deteriorate. One purpose of the present study is also to give an upper validity limit, in terms of the amplitude of the response, of these approximated NNMs, as three different cases are tested.

On the other hand, the proposed method bears a number of advantages. On the theoretical viewpoint, the main advantage of the NNM method is that it relies on the idea of invariance, ensuring proper truncations, as already shown in [10], and in [2] where a full comparison with the POD method is performed. Secondly, as compared to more numerically involved methods as the one by Jiang et al. [13], the reduction process is here quick and easy to use. Finally, the number of NNMs that one must keep in 
the truncation is known beforehand by simply looking at the internal resonances in the linear spectrum. If no internal resonance is present, a single NNM is enough to catch the dynamics. This will be shown in Sections 4 and 5. If internal resonances are present, one has to keep all the NNMs involved in the internal resonance. One key point of the method presented here, relying on normal form theory, is that no extra work is needed to handle the case of internal resonance. In Section 6 concerned with the Flügge shell, as a consequence of the fact that the shell is closed, degenerate eigenvalues are present, giving birth to companion modes, linearly independent but having the same eigenfrequency. Thus 1:1 internal resonance are present, and two NNMs will be kept in order to reduce the system.

\section{Hyperbolic paraboloid panel}

\subsection{Boundary conditions and projection functions}

The HP panel is shown in Fig. 1. The curvilinear axial coordinates are specified by setting $x_{1}=x$, and $x_{2}=y$. The radii of curvature are such that $R_{x}=-R_{y}$.

Classical simply supported boundary conditions at the four edges are assumed, so that:

$v=w=N_{x}=M_{x}=0 \quad$ at $x=0, a$,

$u=w=N_{y}=M_{y}=0 \quad$ at $y=0, b$,

where $N_{x}, N_{y}$ are the normal forces and $M_{x}, M_{y}$ the bending moments per unit length.

The basis functions are respectively

$\phi_{m, n}^{(u)}(x, y)=\cos (m \pi x / a) \sin (n \pi y / b)$,

$\phi_{m, n}^{(v)}(x, y)=\sin (m \pi x / a) \cos (n \pi y / b)$,

$\phi_{m, n}^{(w)}(x, y)=\sin (m \pi x / a) \sin (n \pi y / b)$.

Two non-linear terms $\hat{u}$ and $\hat{v}$ are added to Eqs. (22a) and (22b), respectively, in order to identically satisfy the boundary conditions, as shown in [18].

\subsection{Simulation results}

Numerical simulations have been performed for a HP panel with $a=b=0.1 \mathrm{~m}, R_{x}=-R_{y}=1 \mathrm{~m}$, and thickness $h=1 \mathrm{~mm}$. The material is linear elastic with Young's modulus $E=206.10^{9} \mathrm{~Pa}$, density $\rho=7800 \mathrm{~kg} \mathrm{~m}^{-3}$ and Poisson's ratio $v=0.3$. The response of the HP panel to harmonic excitation in the vicinity of the first eigenfrequency $\omega_{1}$ is numerically computed. The convergence of the solution has been carefully studied in [18] for an excitation amplitude $\tilde{f}$ of $4.37 \mathrm{~N}$ applied at the center of the panel. It has been shown that 22 basis functions were necessary to obtain convergence. More precisely, the generalized coordinates retained for this reference solution are $w_{1,1}, w_{1,3}$, $w_{3,1}, w_{3,3}, u_{1,1}, u_{3,1}, u_{1,3}, u_{3,3}, u_{1,5}, u_{5,1}, u_{3,5}, u_{5,3}, u_{5,5}, v_{1,1}$, $v_{3,1}, v_{1,3}, v_{3,3}, v_{1,5}, v_{5,1}, v_{3,5}, v_{5,3}, v_{5,5}$. The damping parameter

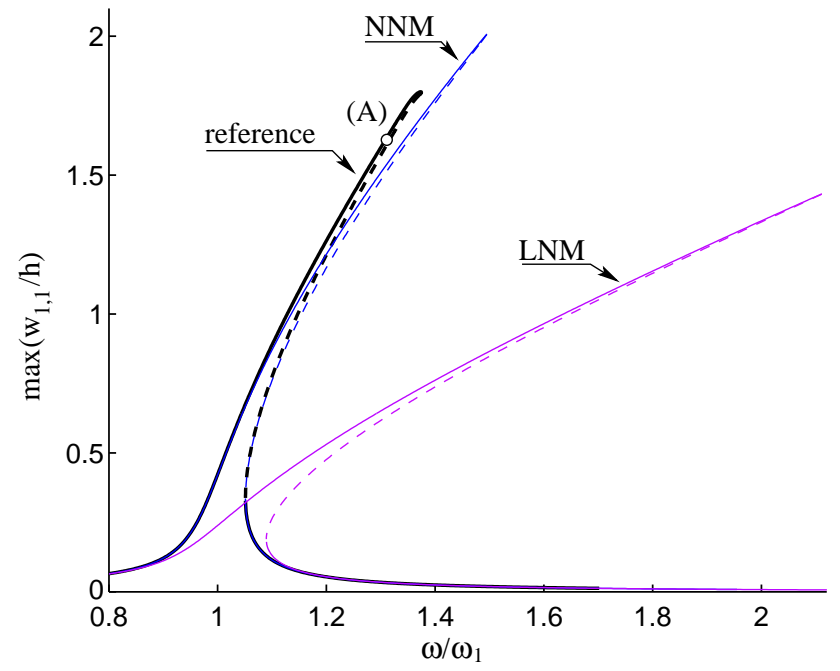

Fig. 2. Frequency-response curve for the HP panel, harmonically excited in the vicinity of the first eigenfrequency $\omega_{1}$. The reference solution is compared to the solution given by keeping a single linear mode (LNM) or a single NNM. The excitation amplitude is $\tilde{f}=4.37 \mathrm{~N}$. Point (A), with $\omega=1.3 \omega_{1}$, is used for time integration, see Fig. 5 .

$\zeta_{p}$ has been set to 0.004 for each mode: $\forall p=1 \ldots$ $22, \zeta_{p}=0.004$.

Fig. 2 shows the frequency-response curve for this reference solution, numerically obtained by a continuation method (pseudo-arclength is used) implemented within the software AUTO [25]. The reference solution with 22 basis functions is compared to two severely reduced-order models, composed of a single oscillator equation. The first one is obtained by keeping in the truncation only the first $\operatorname{LNM}\left(P_{\mathrm{LNM}}=1\right)$. Eq. $(18)$ are restricted to the first one

$$
\begin{aligned}
& \ddot{X}_{1}+2 \zeta_{1} \omega_{1} \dot{X}_{1}+\omega_{1}^{2} X_{1}+g_{11}^{1} X_{1}^{2}+h_{111}^{1} X_{1}^{3} \\
& \quad=F_{1} \cos (\omega t) .
\end{aligned}
$$

Branches of solution are numerically obtained by continuation with AUTO, then the original coordinates are recovered via: $\mathbf{q}=\mathbf{P X}$, where, in $\mathbf{X}$, only the first coordinate $X_{1}$ is different from zero.

The second reduced-order model is obtained by keeping the first NNM: Eq. (20) are truncated by letting $R_{p}=0, \forall p=2 \ldots 22$. The dynamics onto the invariant manifold is then governed by

$$
\begin{aligned}
\ddot{R}_{1} & +2 \zeta_{1} \omega_{1} \dot{R}_{1}+\omega_{1}^{2} R_{1}+\left(h_{111}^{1}+A_{111}^{1}\right) R_{1}^{3}+B_{111}^{1} R_{1} \dot{R}_{1}^{2} \\
& +C_{111}^{1} R_{1}^{2} \dot{R}_{1}=F_{1} \cos (\omega t) .
\end{aligned}
$$

Eq. (24) is solved numerically with AUTO, then one uses Eqs. (19) to come back to the modal coordinates, and finally the matrix of eigenvectors $\mathbf{P}$ allows reconstitution of the amplitudes in the basis of selected projection functions. Thanks to the non-linear nature of the change of variable (19), all the modal amplitudes are non-zero.

Fig. 2 shows the main coordinate $w_{1,1}$, having the most significant response. One can observe that the non-linearity 
is of the hardening type, and that the amplitude of the response, of the order of two times the thickness, is large. The two reduced models have been selected because they share the same complexity: a single oscillator equation is used. Whereas reduction to a single linear mode gives poor result, reduction to a single NNM give a satisfactory result, with a slight overestimation of the maximum vibration amplitude.

Moreover, as shown in Fig. 3, the reduced model composed of a single NNM, thanks to the non-linear change of coordinate, allows recovering all the other coordinates that are not directly excited. Fig. 3 shows the six main coordinates, i.e. the first four coordinates in transverse direction, $w_{1,1}, w_{3,1}, w_{1,3}$ and $w_{3,3}$, as well as the first two longitudinal coordinates $u_{1,1}$ and $v_{1,1}$. It is observed that with the NNM ROM, energy is recovered in all the coordinates, with a good approximation of the original amplitudes. On the other hand, for the model composed of a single linear mode, non-zero amplitudes are recovered only on $w_{1,1}, u_{1,1}$ and $v_{1,1}$, as these three coordinates are linearly coupled to create the first eigenmode described by $X_{1}$ which is simulated. But a vanishing response is found with this LNM ROM for $w_{3,1}, w_{1,3}$ and $w_{3,3}$.

This first result emphasizes the main characteristic of the NNM ROM: the geometrical complexity due to the curvature of the invariant manifold, is first computed in the nonlinear change of coordinates. Once the dynamics reduced to the manifold, a single oscillator equation is sufficient to recover the dynamics. Then, coming back to the original coordinates allows recovering energy onto the slave modes thanks to the non-linear projection.
Fig. 4 shows a representation of the invariant manifold (the first NNM) for the HP panel. The dimension of the phase space is 45 ( 22 oscillator equations with displacement and velocity as independent variables plus the external forcing), and the NNM surface is two-dimensional. A projection in the reduced space $\left(w_{1,1}, \dot{w}_{1,1}, w_{3,1}\right)$ is shown. A trajectory is also represented, which has been computed by numerically integrating the original system described by Eq. (17). The closed orbit represents the true solution, as the reference equations have been used. One can observe that the closed orbit do not fully belong to the invariant manifold. This is the consequence of the two assumptions used to generate the NNM solutions: a third-order asymptotic development is used to approach the invariant manifold, and secondly, a time-invariant manifold is used. As a consequence, the trajectory do not fall completely within the NNM. However a good approximation of the local geometry is provided.

The time solutions for the four most significant coordinates is shown in Fig. 5. Once again, the reference solution is compared to the two reduced models composed of a single linear and non-linear mode. Time integrations have been performed for $\tilde{f}=4.37 \mathrm{~N}$ and $\omega=1.3 \omega_{1}$ (Point (A) in Fig. 2). Whereas the reduction to a single linear mode is not acceptable, the solutions provided by a single NNM are very good. Despite the fact that only one oscillator-equation is simulated, a variety of complex signals are recovered thanks to the non-linear change of coordinates.

The convergence of the solution with an increasing number of LNMs is shown in Fig. 6 for the excitation
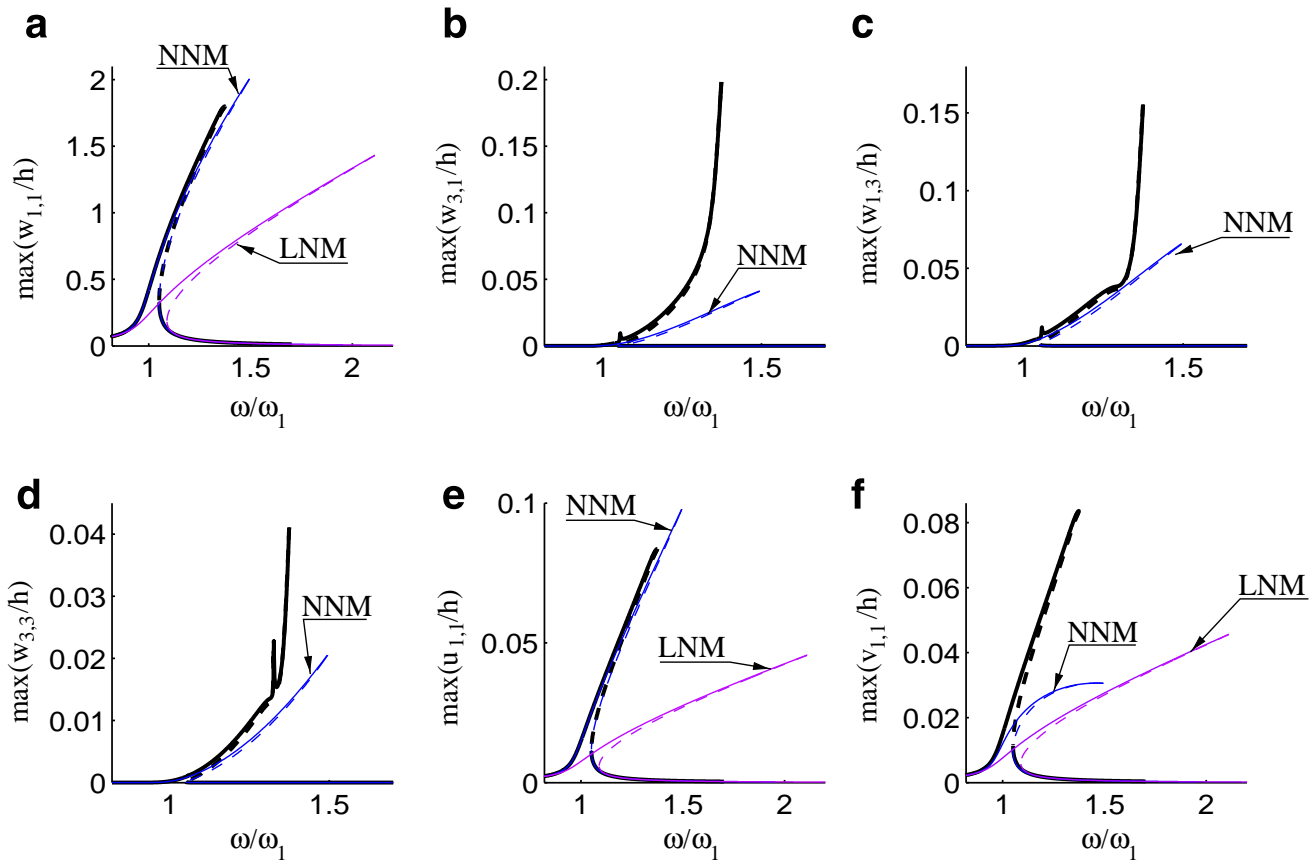

Fig. 3. Maximum amplitude of the response of six generalized coordinates versus excitation frequency, for an excitation amplitude of $\tilde{f}=4.37 \mathrm{~N}$. Reference solution (thick line) is compared to the reduction to a single linear mode (LNM) and a single non-linear mode (NNM): (a) maximum of $w_{1,1}$, (b) maximum of $w_{3,1}$, (c) maximum of $w_{1,3}$, (d) maximum of $w_{3,3}$, (e) maximum of $u_{1,1}$ and (f) maximum of $v_{1,1}$. 


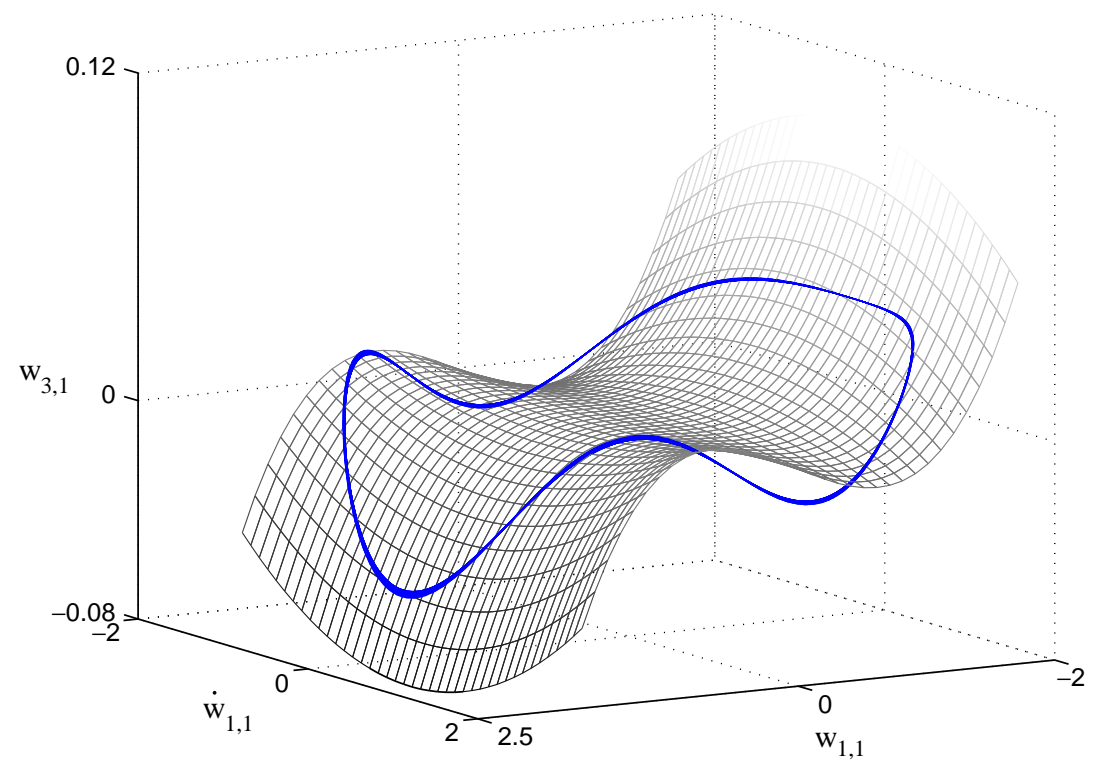

Fig. 4. The invariant manifold (first NNM) for the HP panel in the reduced phase space $\left(w_{1,1}, \dot{w}_{1,1}, w_{3,1}\right)$, calculated with the third-order approximation derived from Eqs. (19). The orbit has been computed by integrating the temporal dynamics of the reference solution, with $\tilde{f}=4.37 \mathrm{~N}$ and $\omega=1.3 \omega_{1}$.
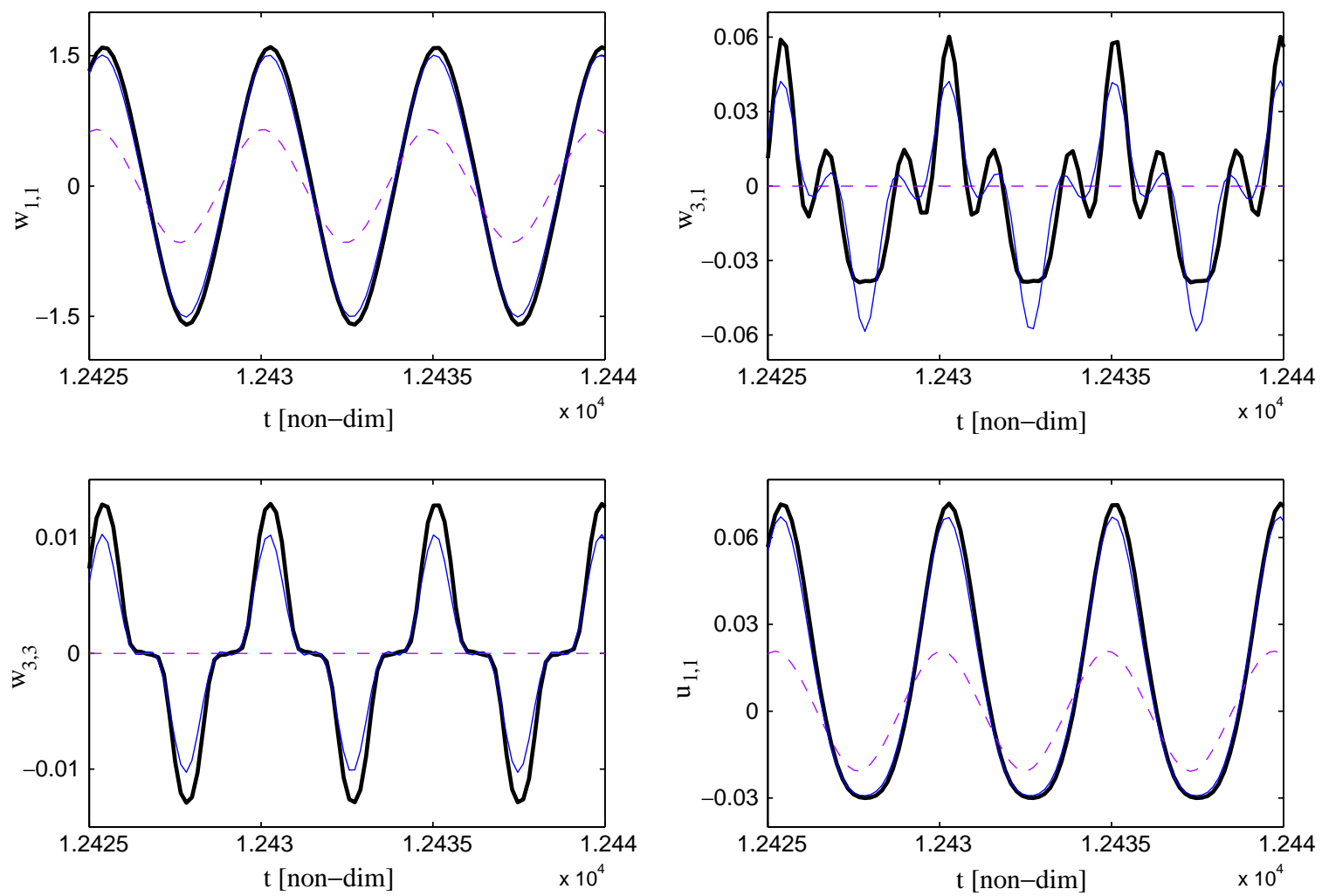

Fig. 5. Time-domain response of four generalized coordinates of the HP panel, excitation frequency $\omega=1.3 \omega_{1}$, amplitude $\tilde{f}=4.37 \mathrm{~N}$. Reference solution (thick line) is compared to the NNM solution (thin line), and the LNM solution (dashed line).

amplitude of $4.37 \mathrm{~N}$. It is found that the convergence is very slow: 15 LNMs are necessary to obtain an acceptable solution. The solution with $11 \mathrm{LNMs}$ is qualitatively different from the converged solution with a strange loop appearing in the frequency response, and is thus not acceptable. Hence a very slow convergence with respect to increasing $P_{\mathrm{LNM}}$ is found, and using the linear normal modes is not very favourable as compared to the projection functions used. On the other hand, it has been found that increasing the number of NNMs kept in the truncation in Eq. (20) do not change anything in the solution: the added NNMs have been found to stay with constant neglectable 


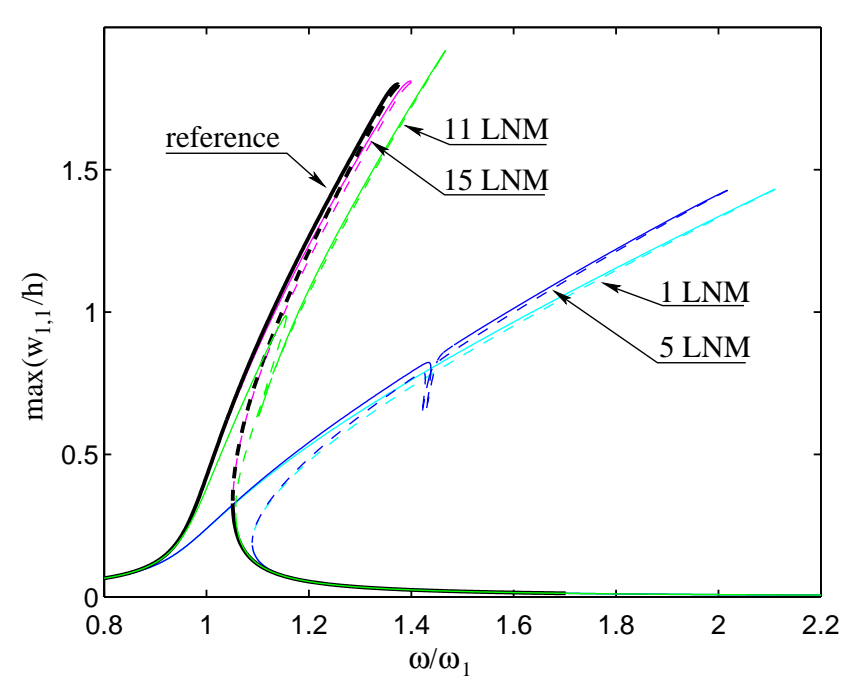

Fig. 6. Maximum amplitude response of $w_{1,1}$ versus excitation frequency for $\tilde{f}=4.37 \mathrm{~N}$, showing the convergence of the solution when increasing $P_{\mathrm{LNM}}$. Reference solution (22 basis functions) is compared to truncations with 1 linear mode, 5 LNM, 11 LNM and 15 LNM.

amplitude, and the same solution is found as the one obtained with a single NNM. This is a logical consequence of the invariance property of the NNMs. Hence the solution with a single NNM seems to be the best ROM possible. The only way to improve the results found here is not in increasing the number of NNMs, but in overshooting the two limitations of the present approximation used for generating the NNMs.

Finally, the robustness of the ROMs with respect to increasing the amplitude of the forcing, is studied. Fig. 7 shows the results obtained for a lower excitation amplitude: $\tilde{f}=2.84 \mathrm{~N}$, and for a larger one: $\tilde{f}=6.62 \mathrm{~N}$. For $\tilde{f}=2.84 \mathrm{~N}$, the result given by the NNM ROM is almost perfectly coincident with the reference solution obtained with 22 basis function, whereas the model with a single linear mode give unacceptable results. For the larger ampli- tude, $\tilde{f}=6.62 \mathrm{~N}$, the result deteriorates for the NNMreduced model, which is not able to catch the saturation loop found by the reference solution at the top of the frequency-response curve. The observation of the other coordinates (not shown for the sake of brevity) shows that this loop reflects the fact that most of the energy is, at this point, absorbed by the higher modes, the amplitude of which significantly and abruptly increase. This subtil change in the dynamics of the system is not caught by the reduced model, which overpredict the maximum amplitude.

As a conclusion on the HP panel, the dynamics has been reduced from 22 dofs to a single NNM. Results shows that the reduction, computed with an asymptotic expansion to approach the invariant manifold, gives very good results for vibration amplitudes up to 1.5 times the panel thickness $h$. Beyond this value, the two approximations used for generating the ROM do not hold anymore. On the other hand, using truncations with LNMs did not allow substantial improvement as compared to the selected basis functions used for discretizing the problem.

\section{Circular cylindrical panel}

\subsection{Boundary conditions and projection functions}

The CC panel is shown in Fig. 8. The coordinates used to describe the geometry are $x_{1}=x$ and $x_{2}=\theta$, where $(x, \theta)$ are the cylindrical coordinates shown in Fig. 8. As a consequence, the radii of curvature are such that $R_{1}=R_{x}=\infty$, and $R_{2}=R$. The angular span is $\alpha$, length is $a$ and thickness $h$. The selected boundary conditions are:

$$
\begin{aligned}
& w=N_{x}=M_{x}=0, \quad N_{x, y}=0, \quad \text { at } x=0, a, \\
& w=N_{y}=M_{y}=0, \quad N_{y, x}=0, \quad \text { at } y=0, b,
\end{aligned}
$$

where $y=R \theta$ and $b=R \alpha$. They modelize a restrained condition in transverse direction with fully free in-plane displacements.
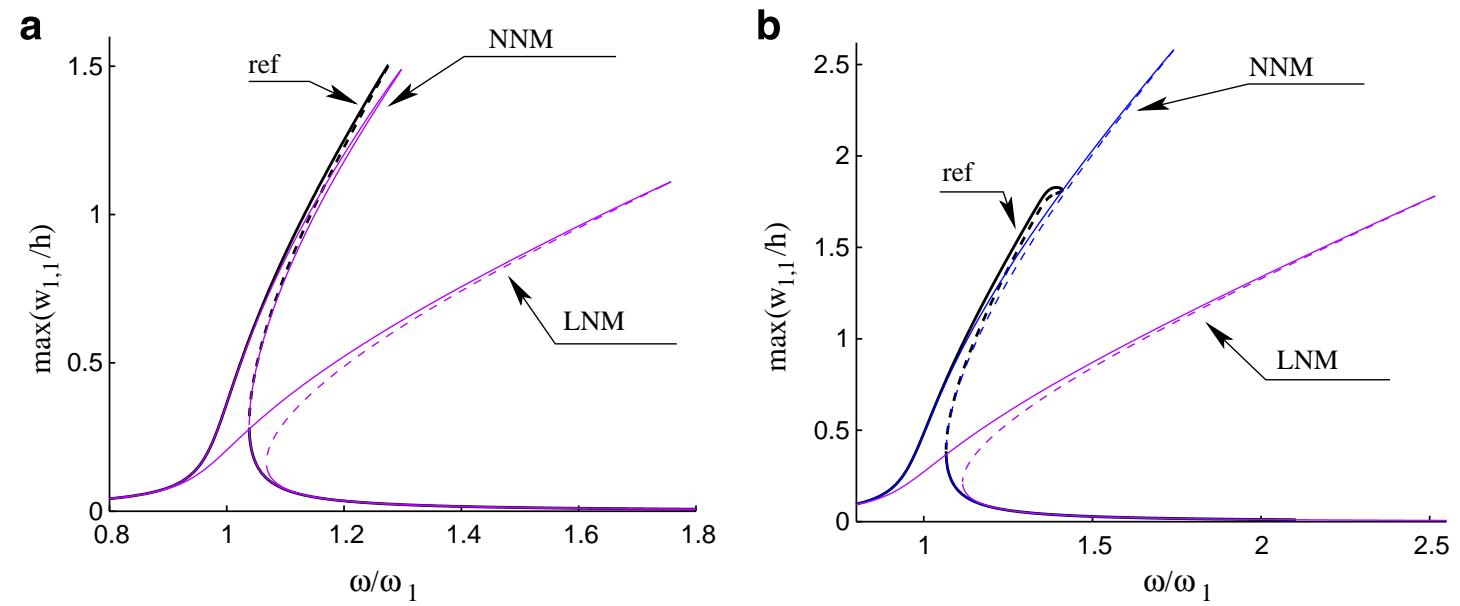

Fig. 7. Frequency-response curve for (a) $\tilde{f}=2.84 \mathrm{~N}$, and (b) $\tilde{f}=6.62 \mathrm{~N}$. Reference solution (ref) is compared to truncations with a single linear mode (LNM) and a single non-linear mode (NNM). 


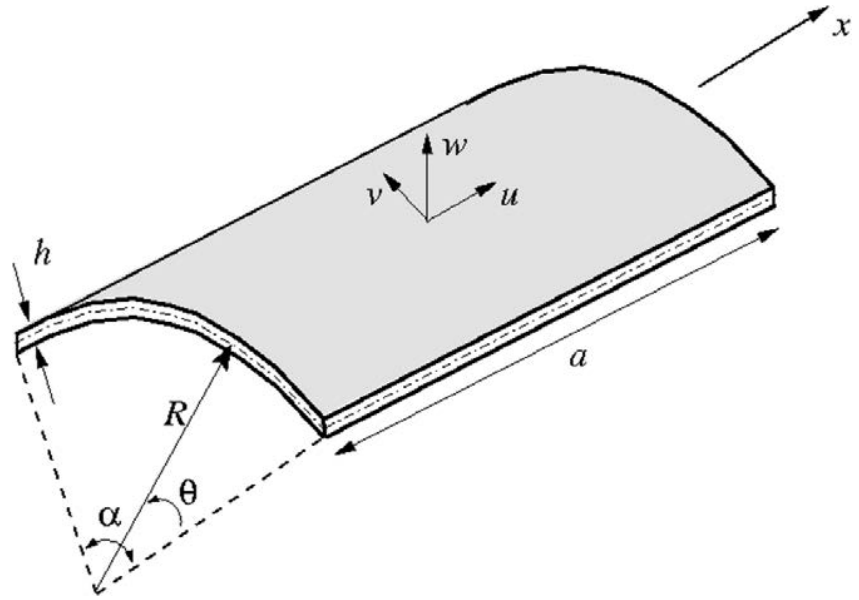

Fig. 8. Geometry and coordinates axis for the circular cylindrical panel.

The basis functions are respectively

$\phi_{m, n}^{(u)}(x, y)=\cos (m \pi x / a) \cos (n \pi \theta / \alpha)$,

$\phi_{m, n}^{(v)}(x, y)=\cos (m \pi x / a) \cos (n \pi \theta / \alpha)$,

$\phi_{m, n}^{(w)}(x, y)=\sin (m \pi x / a) \sin (n \pi \theta / \alpha)$.

Two non-linear terms $\hat{u}$ and $\hat{v}$ are added to Eqs. (26a) and (26b), respectively, in order to identically satisfy the boundary conditions, as shown in $[19,28]$.

\subsection{Simulation results}

Numerical simulations have been performed for a specific panel with $a=0.1 \mathrm{~m}, R=1 \mathrm{~m}$ and $h=1 \mathrm{~mm}$. The angular span is $\alpha=0.1 \mathrm{rad}$, so that the panel length equals the circumferential width. Material properties are identical to that used for the HP panel so that $E, \rho$ and $v$ are unchanged. The $\mathrm{CC}$ panel response to harmonic excitation in the vicinity of the first eigenfrequency is studied. The convergence of the reference solution has been carefully checked in $[19,28]$, for an excitation amplitude of $\tilde{f}=4.4 \mathrm{~N}$ applied at the center of the panel. It has been found that the minimal number of basis functions should be 19. This solution will be referred to as the reference solution; the selected basis functions are: $w_{1,1}, w_{1,3}, w_{3,1}, w_{3,3}$, $u_{1,0}, u_{1,2}, u_{1,4}, u_{3,0}, u_{3,2}, u_{3,4}, v_{0,1}, v_{2,1}, v_{4,1}, v_{0,3}, v_{2,3}, v_{4,3}, v_{0,5}$, $v_{2,5}, v_{4,5}$. Damping coefficient $\zeta_{p}$ has been set equal to 0.004 for each generalized coordinate.

Fig. 9 shows the response of the main coordinate $w_{1,1}$ for an excitation amplitude of $\tilde{f}=2.2 \mathrm{~N}$. The reference solution, obtained with 19 dofs, is contrasted to two ROMs composed of a single dof: a single LNM and a single NNM. The results are comparable to those obtained for the HP panel. The response of the panel is of the hardening type. The restriction to a single linear mode is a too severe truncation that leads to a huge overestimation of the hardening behaviour of the panel. On the other hand, the response provided by the NNM ROM is quasi-coincident with the reference solution.

As already mentioned for the HP panel, the NNM ROM allows recovering non-directly excited coordinates thanks to the non-linear relationship between the original (modal) coordinates and the master mode that is retained for simulation. Comparison of other coordinates are shown in Fig. 10. A particular feature of this model is the weak coupling observed between the coordinates. This is revealed here by the very small values of maximum amplitude of the non-directly excited coordinates, recovered via the changes of variables: they are an order of magnitude less compared to the HP panel. On the geometrical viewpoint, it means that the invariant manifold is rather flat. The non-linearity is here more focused on the selfexciting terms present in the normal form than in the coupling amongst eigenmodes, which are treated by the curvature of the NNM manifold. The convergence of the reduced systems have also been tested. As in the precedent case of the HP panel, a very slow convergence with increasing the number of linear modes have been found: 15 LNMs were necessary in order to recover the original results. Other more severe truncations with a number of linear modes less or equal than 8 gave unacceptable results.

Fig. 11 shows the comparison between the NNM ROM and the reference solution, for an excitation amplitude of

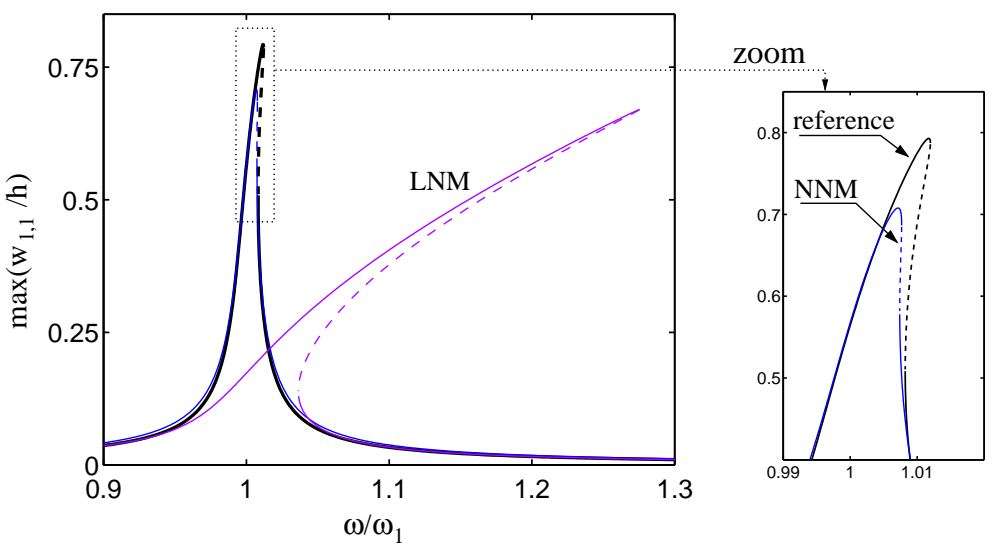

Fig. 9. Maximum amplitude of coordinate $w_{1,1}$ versus excitation frequency for the CC panel, $\tilde{f}=2.2 \mathrm{~N}$. NNM solution is quasi-coincident with the reference solution. Truncated solution with a single linear mode (LNM) is also shown. 

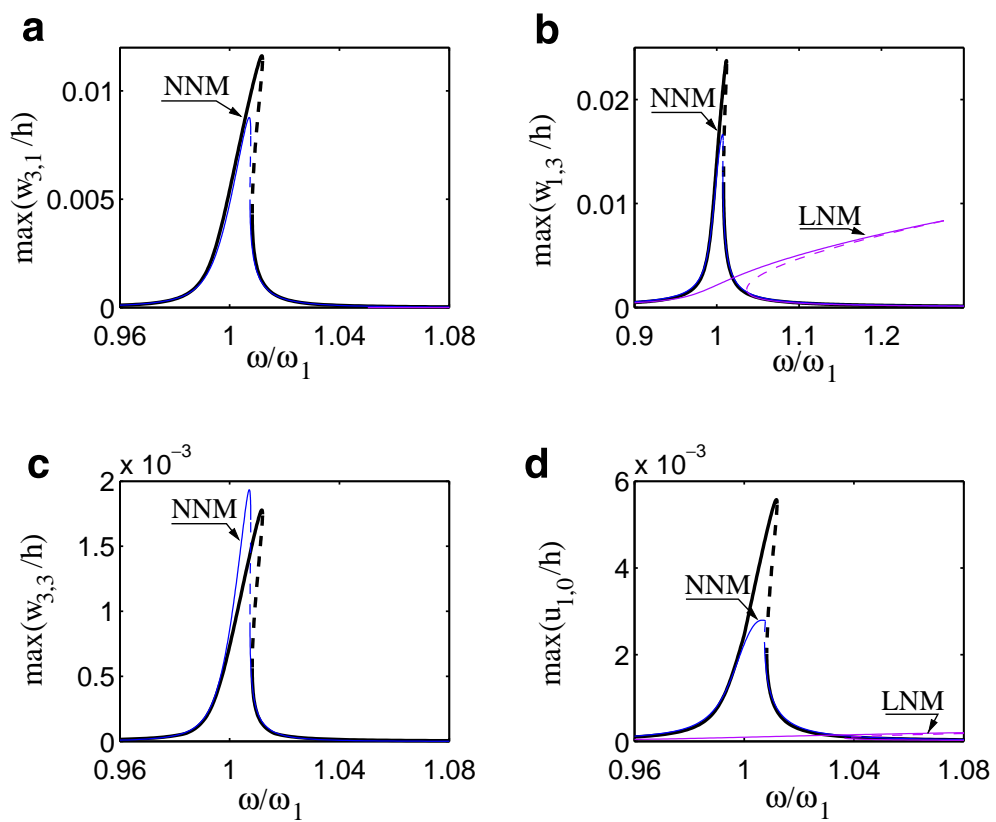

Fig. 10. Maximum amplitude versus excitation frequency, CC panel, $\tilde{f}=2.2 \mathrm{~N}$. Reference solution (thick line) is compared to single NNM and single LNM solutions. Four non-directly excited coordinates are shown: (a) $w_{3,1}$, (b) $w_{1,3}$, (c) $w_{3,3}$ and (d) $u_{1,0}$.
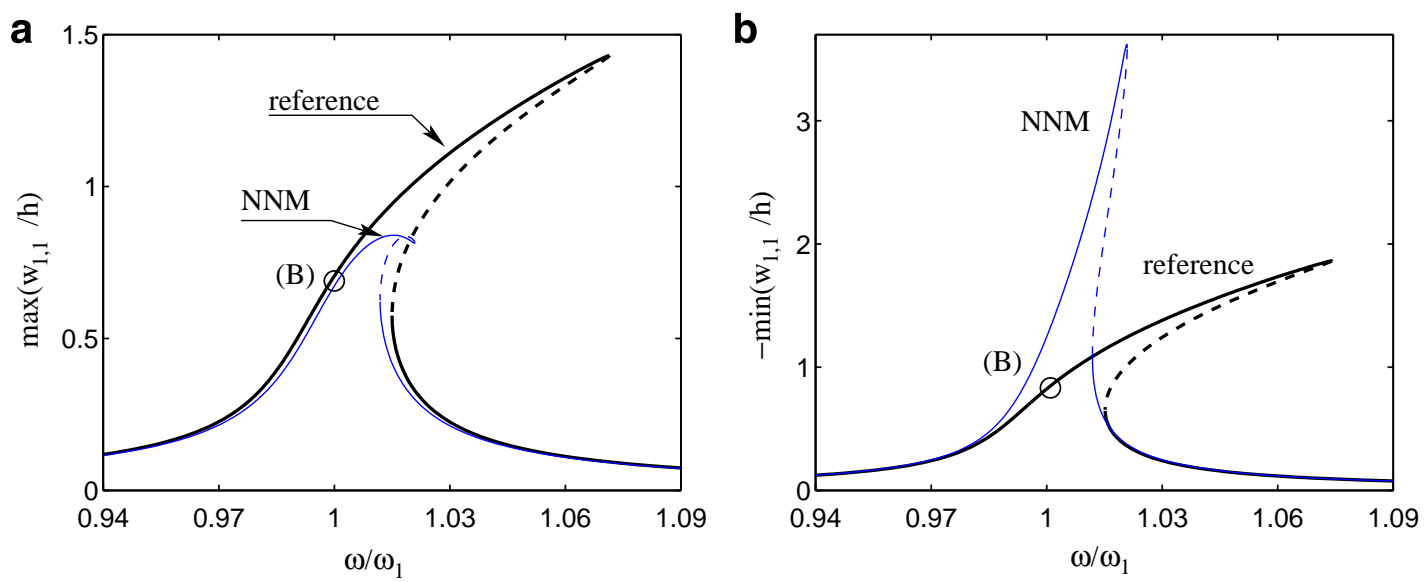

Fig. 11. Frequency-response curve for coordinate $w_{1,1}$, excitation amplitude of $4.4 \mathrm{~N}$ : (a) maximum amplitude response (outwards deflection) and (b) minimum amplitude response (inwards deflection). Reference solution is compared to single NNM truncation. Point $(\mathrm{B})$, with $\omega=\omega_{1}$, is used for time integration, see Fig. 12.

4.4 N. The results provided by the single linear mode ROM has not been plotted as it was completely unacceptable. As a consequence of the weak coupling amongst generalized coordinates detected before, the NNM simulation fails in recovering the resonance curve for this level of excitation. It can be explained by the fact that the NNM ROM, in its third-order asymptotic approximation used here, has the primary ability to catch very well the coupling amongst linear modes through the non-linear change of coordinates. As these couplings are here weak, the ROM is rapidly limited by its third-order expansion, that shows some difficulties to recover the non-linearity exhibited in this case.
Moreover, the reference simulation shows an important asymmetry between outwards displacements (Fig. 11a with the maximum amplitude) and inwards displacements (Fig. 11b with the minimum amplitude). This asymmetry is caught by the NNM ROM but dramatically increased. This, once again, shows that, in this case, the third-order expansion is not enough to have a good approximation of the dynamics.

Direct integration of the equations of motion for the reference model is compared to the single LNM and NNM time simulations in Fig. 12, for this large value of excitation $\tilde{f}=4.4 \mathrm{~N}$, and $\omega=\omega_{1}$. As predicted by the frequency- 

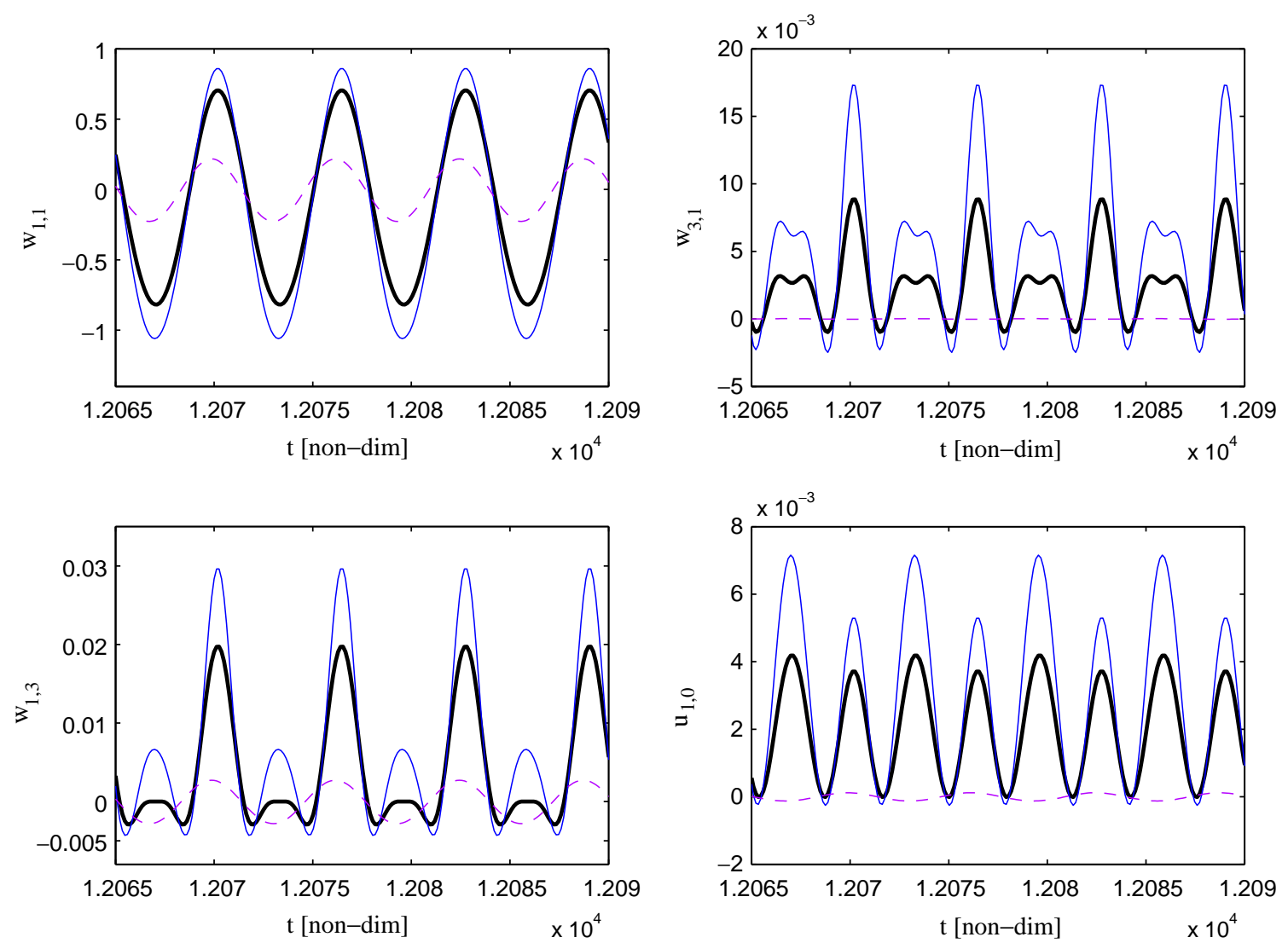

Fig. 12. Time-domain response of four generalized coordinates of the CC panel, excitation frequency $\omega=\omega_{1}$, amplitude $\tilde{f}=4.4 \mathrm{~N}$. Reference solution (thick line) is compared to the NNM solution (thin line), and the LNM solution (dashed line).

response curve, the asymmetry is overestimated by the NNM ROM. However, as in the case of the HP panel, a large variety of non-linear temporal signals are well recovered, whereas a single oscillator-equation is simulated.

Finally, in the case of the CC panel, the NNM ROM gives good results for vibration amplitudes up to $0.9 h$.

\section{Closed circular cylindrical shell}

\subsection{Boundary conditions and projection functions}

The third example is a closed, empty shell of length $a$. The geometry and coordinate system is deduced from the CC panel by selecting $\alpha=2 \pi$ for the angular span. Whereas the strain-displacement relationship for the two precedent example was given by Donnell's non-linear theory with in-plane inertia, Flügge-Lur'e-Byrne non-linear theory is used here. The reader is referred to $[17,23,24]$ for a more thorough description of the kinematics.

The boundary conditions are simply supported at the two ends $x=0, a$

$w=M_{x}=v=N_{x}=0, \quad$ at $x=0, a$,

where $M_{x}$ and $N_{x}$ are respectively the bending moment and the axial force per unit length.

The response of the shell to harmonic forcing in the vicinity of mode $(m, n)$, where $m$ is the number of longitu- dinal half-waves, and $n$ of circumferential waves, is considered. Based on past studies, where a detailed convergence study was considered for mode $(1,5)$ (see e.g. [17] and references therein), the minimal expansion used for discretizing the shell has been found to be the following:

$$
\begin{aligned}
u(x, \theta, t)= & \sum_{k=1}^{2}\left[u_{1,5 k, c}(t) \cos (5 k \theta)+u_{1,5 k, s}(t) \sin (5 k \theta)\right] \cos \left(\lambda_{1} x\right) \\
& +\sum_{m=1}^{2} u_{2 m-1,0}(t) \cos \left(\lambda_{2 m-1} x\right)+\hat{u}, \\
v(x, \theta, t)= & \sum_{k=1}^{2}\left[v_{1,5 k, c}(t) \sin (5 k \theta)+v_{1,5 k, s}(t) \cos (5 k \theta)\right] \sin \left(\lambda_{1} x\right) \\
& +\left[v_{3,10, c}(t) \sin (10 \theta)+v_{3,10, s}(t) \cos (10 \theta)\right] \sin \left(\lambda_{3} x\right),
\end{aligned}
$$

$$
\begin{aligned}
w(x, \theta, t)= & {\left[w_{1,5, c}(t) \cos (5 \theta)+w_{1,5, s}(t) \sin (5 \theta)\right] \sin \left(\lambda_{1} x\right) } \\
& +\sum_{m=1}^{2} w_{2 m-1,0}(t) \sin \left(\lambda_{2 m-1} x\right),
\end{aligned}
$$

where $\lambda_{m}=\frac{m \pi}{a}$, and $\hat{u}$ is a non-linear term added to satisfy exactly the boundary condition $N_{x}=0$. Because of the circumferential symmetry, degenerate modes appear in the structure; they are here denoted with the additional subscript $c$ or $s$, indicating if the generalized coordinates is associated with a cos or sin function in the angular coordinate $\theta$ for $w$. This expansion gives the reference solution, 
which is obtained here with 16 generalized coordinates. One can observe that the basis functions are in fact the eigenmodes of the empty shell for the transverse component.

The point excitation considered is located at a node of the mode $(1,5, s)$. Consequently, mode $(1,5, c)$ is called the driven mode, and mode $(1,5, s)$, which is not directly excited by the external load, is the companion mode. The damping coefficient $\zeta_{p}$ is selected such that $\zeta_{p}=\zeta=0.001$ for each asymmetric modes, whereas for axisymmetric modes (present in the truncation via $w_{1,0}, w_{3,0}, u_{1,0}$ and $\left.u_{3,0}\right)$ we have: $\zeta_{1,0}=\frac{\omega_{1,0}}{\omega_{1,5}} \zeta$ and $\zeta_{3,0}=\frac{\omega_{3,0}}{\omega_{1,5}} \zeta$.

\subsection{Simulation results}

Numerical simulations have been performed for a shell whose geometric dimensions are: $a=520 \mathrm{~mm}, R=$
$149.4 \mathrm{~mm}, \quad h=0.519 \mathrm{~mm}$. Material properties are: $E=1.98 \times 10^{11} \mathrm{~Pa}, \rho=7800 \mathrm{~kg} \mathrm{~m}^{-3}, v=0.3$. As a consequence of the circumferential symmetry and the appearance of degenerate modes, 1:1 internal resonances are present in the system. More particularly in the present case, the driven mode and the companion mode are 1:1 internally resonant. Hence the minimal model that could capture the dynamics should contain at least two oscillator equations. This minimal model is given by keeping the first two NNMs, corresponding respectively to the continuation of driven and companion modes, in the same way as the truncation realized on a fluid-filled cylindrical shell described by Donnell shallow-shell theory, see $[15,2]$.

In the remainder of the study, the reference solution obtained with 16 basis functions will be compared to the solution given by the reduced model composed of 2 NNMs. The comparison with a truncation of linear modes,
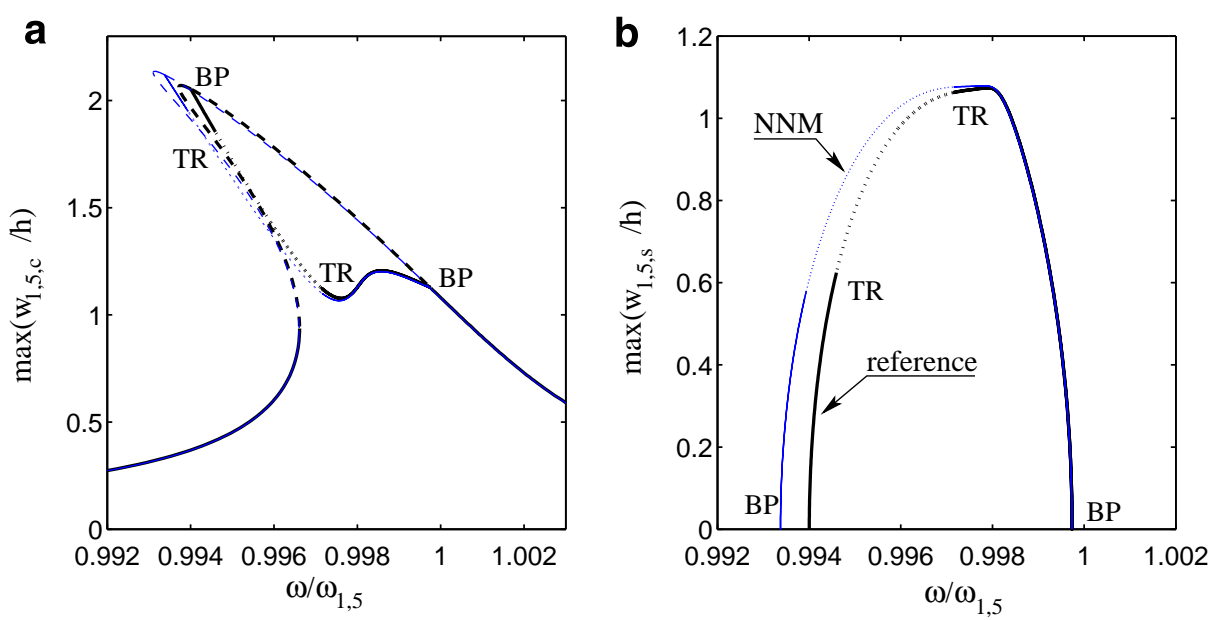

Fig. 13. Maximum amplitude of vibration versus excitation frequency for the Flugge shell, with an excitation amplitude of 2 N. Comparison of reference solution (thick line) to the ROM composed of two NNMs (NNM): (a) maximum amplitude of the driven mode $w_{(1,5, c)}$ and (b) maximum amplitude of the companion mode $w_{(1,5, s)}$. BP: branch points on the single-mode branch of solution, leading to coupled solutions (branch 2). TR: Neimarck-Sacker (Torus) bifurcation points leading to quasi-periodic solutions.
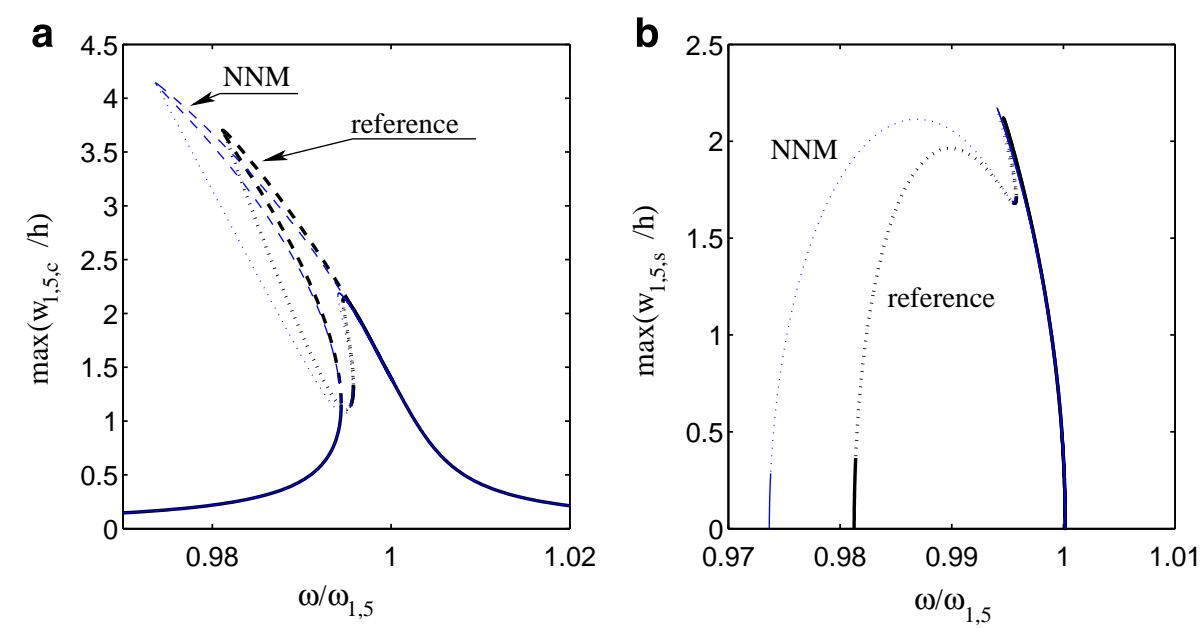

Fig. 14. Maximum amplitude of vibration versus excitation frequency for the Flugge shell, $\tilde{f}=4 \mathrm{~N}$. Comparison of reference solution (thick line) to the ROm composed of two NNMs (NNM): (a) maximum amplitude of the driven mode $w_{(1,5, c)}$ and (b) maximum amplitude of the companion mode $w_{(1,5, s)}$. 
as in the precedent cases, will not be shown for the following reason. Once again, the convergence of the solution with the number of linear modes was very slow. Moreover, all the truncations tried with an increasing number of linear modes predicted a hardening-type non-linearity, except the solution with 16 linear modes which recovers the original solution with a softening-type non-linearity for the driven mode. Hence the minimal number of linear modes is also 16 , and no other truncation is acceptable.

Fig. 13 shows the comparison between the reference solution and the reduced model composed of two NNMs, for an excitation amplitude $\tilde{f}=2 \mathrm{~N}$. The solution is composed of two branches. The first branch corresponds to the single-mode response: the companion mode has a zero amplitude. This branch displays a softening-type non-linearity, and shows two branch points (BP), from which the second branch arises. This second branch corresponds to coupled solutions where both driven and companion mode have a non-zero amplitude. Following branch 2, a bifurcation occurs via torus (Neimarck-Sacker) bifurcations (TR), leading to a quasi-periodic regime.

As shown in Fig. 13, the reduced model perfectly recovers all the details of this complicated bifurcation diagram. A slight overestimation of the softening non-linearity is detected, but all the bifurcation points, as well as their nature, are perfectly recovered. In contrast, no other solution with a truncation with the linear modes, was able to catch the softening type non-linearity. This excellent result confirms earlier investigations led on a fluid-filled cylindrical shell, see [15,2].

The robustness of the ROM is tested by increasing the external forcing amplitude up to $\tilde{f}=4 \mathrm{~N}$. The corresponding frequency-response is shown in Fig. 14. Despite an increase in the overestimation of the maximum amplitude, all the bifurcation points are once again recovered. From these studies, it can be concluded that the ROM is reliable for vibration amplitudes up to $3 h$.

\section{Discussion}

Reduction methods is a central question in the simulation of vibrating structures. Many different methods have been proposed in the past, one of the most popular being the Proper Orthogonal Decomposition (POD) method [35,29]. In this paper, the NNMs of the unforced structure are tested as basis functions for reducing the dynamics for shell models with harmonic forcing.

On the computational viewpoint, one of the main drawback of the present method is the number of coefficients that have to be computed for obtaining the reduced model. The main task consists in the computation of all the linear $\left\{z_{i}^{p}\right\}_{p, i=1 \ldots P}$, quadratic $\left\{z_{i, j}^{p}\right\}_{p, i, j=1 \ldots P}$ and cubic $\left\{z_{i, j, k}^{p}\right\}_{p, i, j, k=1 \ldots P}$ coupling coefficients appearing in Eq. (17). This can be seen as an extra work as compared to other methods, e.g. discretization based on finite elements procedures, where these coefficients are generally not computed for solving out temporal response for instance. However, in the context of this study where one is interested with frequency-response curves with bifurcation points, this step is necessary. It can also be remarked that the computation of these coefficients are here realized on the basis of analytical developments, however they can easily be implemented within a finite-element based discretization. The second family of coefficients that have to be computed are those from the non-linear change of coordinates defined by Eqs. (19). However the numerical burden associated with this step is limited, so that this does not appear as a limiting factor of the method. Moreover, all these computations are made once and for all, and for the unforced structure, so that these can be seen as off-line calculations, that have not to be repeated for computing other responses. This is of course an advantage as compared to the POD method.

An assessment of the computational burden associated with the numerical results shown in previous section is given in Table 1. The computational times are only indicatory, as they strongly depend on the processor and on numerous parameters that can be tuned in a typical AUTO-run. Moreover, the computation of solution branches with AUTO depends on the complexity of the solution, thus the computational time also depends on the amplitude of the forcing. These values are here given in order to better quantify the gain in using ROMs in typical situations, which is very important. A typical AUTOrun for computing a single-dof frequency-response curve is of the order of $30 \mathrm{~s}$ (values given for the LNM ROM). For the NNM ROM, about $3 \mathrm{~min}$ are spent in order to compute the non-linear change of coordinates, that are added to the $30 \mathrm{~s}$ of the AUTO-run. However, as the non-linear change of coordinates is computed once and for all, the single-dof NNM ROM can be used for comput-

Table 1

Comparisons of accuracy and computational times for the three selected models

\begin{tabular}{llll}
\hline & Reference & LNM & NNM \\
\hline HP Panel & 22 dofs & 1 dof & 1 dof \\
Comp. time & $1 \mathrm{~h} 56 \mathrm{~min}$ & $36 \mathrm{~s}$ & $4 \mathrm{~min} 47 \mathrm{~s}$ \\
Validity limits & $/$ & $0.1 h$ & $1.5 h$ \\
CC Panel & 19 dofs & 1 dof & 1 dof \\
Comp. time & $1 \mathrm{~h} 28 \mathrm{~min}$ & $35 \mathrm{~s}$ & $3 \mathrm{~min} 43 \mathrm{~s}$ \\
Validity limits & $/$ & $0.1 h$ & $0.9 h$ \\
Flügge shell & 16 dofs & 2 dofs & 2 dofs \\
Comp. time & $1 \mathrm{~h} 37 \mathrm{~min}$ & $/$ & $2 \mathrm{~min} 48 \mathrm{~s}$ \\
Validity limits & $/$ & $0.1 h$ & $3 h$ \\
\hline
\end{tabular}

The computational time is defined as the time needed for computing a typical frequency-response curves. For the reference solution, this includes the time spent during an AUTO-run. For the LNM ROM, to the time spent in an AUTO-run is added the time spent in the linear change of coordinates (application of $\mathbf{P}$ ). For the NNM ROM, the time spent for computing and applying the non-linear change of coordinate is also added. The amplitude of the forcing used for this table is: $4.37 \mathrm{~N}$ for the $\mathrm{HP}$ panel, $4.4 \mathrm{~N}$ for the $\mathrm{CC}$ panel, and $2 \mathrm{~N}$ for the Flügge shell. Computations have been realized on a standard PC with a Pentium IV processor working at $2.4 \mathrm{GHz}$. Computational time associated to the Flügge shell reduced to two LNMs is not mentioned as the model has not been able to recover to two solution branches. 
ing any solution, so that the computational time indicated in the NNM column must be understood as an upper limit. From Table 1, one can see that the computation times obtained with the NNM ROMs have been reduced by a significant factor, ranging from 23 (HP panel) to 32 (Flügge shell).

The upper validity limit has also been estimated in each case by increasing the amplitude of the forcing. No validity limits is indicated for the reference solution. However, if larger values of the forcing would have been considered, the convergence study with the projection functions should have been done once again. The reduction to a single LNM generally provided poor results, and once the value of 0.1 times the thickness $h$ is reached, the reduction to a single LNM is not acceptable. For the single NNM ROM, this value is much larger. It has been found that the NNM ROM was very accurate for amplitude up to $1.5 h$ for the HP panel, $0.9 h$ for the $\mathrm{CC}$ panel, and $3 h$ for the Flügge shell. To these three cases, the case of the water-filled circular cylindrical shell studied in [2] can be added, where the accuracy has been estimated to $2.5 h$.

Finally, one can also remark that the present reduction method is particularly efficient for harmonically forced responses, as the motion is confined in the vicinity of the NNMs. However, for other dynamical behaviour (e.g. free vibration of response to white noise), the benefit in using NNMs instead of LNMs is questionable. Due the dynamical characteristics, it is not awaited, in these cases, to obtain as good results as those presented here for forced responses.

Further research in the area of model reduction with NNMs will consist in application of the present method to structures discretized with finite elements procedures. As shown in this study, the method can handle different kinematics, and thus can be interfaced with a finite elements method. Overcoming the limitation of the asymptotic development is also the key to a reliable solution that is not amplitude-dependent.

\section{Conclusion}

The non-linear response to harmonic forcing in the vicinity of the first eigenfrequencies of three shell structures have been studied. Particular attention has been paid to the derivation of reduced-order models (ROMs), that could be able to describe with accuracy the non-linear frequencyresponse curves. Linear normal modes and non-linear normal modes computed by an asymptotic approach have been used, for three selected systems: a hyperbolic paraboloid panel, a circular cylindrical panel and a closed empty circular cylindrical shell.

For the first two cases, the reduction to a single NNM has been possible, showing very good results for moderate vibration amplitude. On the other hand, it has been observed that using the LNMs did not allow for a substantial improvement of the results, as compared to the first expansion functions used for discretizing the panels. A sin- gle LNM gives unacceptable results, and the linear convergence was very slow in the two cases. Using NNMs thus appears as the best solution for reducing the system. In the third case, a 1:1 internal resonance was present in the system, so that the minimal model was composed of two NNMs, and showed very good results in recovering every bifurcations points as well as the nature of the dynamical regimes.

However, the NNM ROMs have been computed with two approximations: a third-order asymptotic development is used to approach the invariant manifold, and timeinvariant NNM is used whereas a time-dependent one should be used to recover the dependence introduced by the external forcing. Hence, for large amplitude of vibrations, the results deteriorate.

As a conclusion, one can note that the present reduction method bears a number of advantage, as it is quick and easy-to-use, and overcomes the problems of internal resonances without extra work. Its reliability can be said to be very good up to $h$ for shell structures, and generally can give good results up to $2 h$ except in the case where the coupling between the modes is weak. To overcome this limitation, the two approximations presently used for generating the NNMs must be revised.

\section{References}

[1] S.W. Shaw, C. Pierre, Non-linear normal modes and invariant manifolds, J. Sound Vib. 150 (1) (1991) 170-173.

[2] M. Amabili, C. Touzé, Reduced-order models for non-linear vibrations of fluid-filled circular cylindrical shells: comparison of POD and asymptotic non-linear normal modes methods, J. Fluids Struct. 23 (6) (2007) 885-903.

[3] G. Kerschen, J.-C. Golinval, A.F. Vakakis, L.A. Bergman, The method of proper orthogonal decomposition for dynamical characterization and order reduction of mechanical systems: an overview, Nonlinear Dynam. 41 (1-3) (2005) 147-169.

[4] R. Sampaio, C. Soize, Remarks on the efficiency of POD for model reduction in non-linear dynamics of continuous elastic systems, Int. J. Numer. Methods Engrg. 72 (1) (2007) 22-45.

[5] P. Krysl, S. Lall, J.E. Marsden, Dimensional model reduction in nonlinear finite element dynamics of solids and structures, Int. J. Numer. Methods Engrg. 51 (2001) 479-504.

[6] S.W. Shaw, C. Pierre, Normal modes for non-linear vibratory systems, J. Sound Vib. 164 (1) (1993) 85-124.

[7] L. Jézéquel, C.-H. Lamarque, Analysis of non-linear dynamical systems by the normal form theory, J. Sound Vib. 149 (3) (1991) 429 459

[8] A.F. Vakakis, L.I. Manevich, Yu.V. Mikhlin, V.N. Pilipchuk, A.A. Zevin, Normal Modes and Localization in Non-linear Systems, Wiley, New-York, 1996.

[9] W. Lacarbonara, G. Rega, A.H. Nayfeh, Resonant non-linear normal modes. Part I: Analytical treatment for structural one-dimensional systems, Int. J. Non-Linear Mech. 38 (6) (2003) 851-872.

[10] C. Touzé, O. Thomas, A. Chaigne, Hardening/softening behaviour in non-linear oscillations of structural systems using non-linear normal modes, J. Sound Vib. 273 (1-2) (2004) 77-101.

[11] I.V. Andrianov, Asymptotic construction of nonlinear normal modes for continuous systems, Non-linear Dynam. 51 (1-2) (2008) 99-109.

[12] E. Pesheck, C. Pierre, S.W. Shaw, A new Galerkin-based approach for accurate non-linear normal modes through invariant manifolds, J. Sound Vib. 249 (5) (2002) 971-993. 
[13] D. Jiang, C. Pierre, S.W. Shaw, Nonlinear normal modes for vibratory systems under harmonic excitation, J. Sound Vib. 288 (45) (2005) 791-812.

[14] R. Arquier, S. Bellizzi, R. Bouc, B. Cochelin, Two methods for the computation of nonlinear modes of vibrating systems at large amplitudes, Comput. Struct. 84 (24-25) (2006) 1565-1576.

[15] C. Touzé, M. Amabili, Non-linear normal modes for damped geometrically non-linear systems: application to reduced-order modeling of harmonically forced structures, J. Sound Vib. 298 (4-5) (2006) 958-981.

[16] C. Touzé, O. Thomas, Non-linear behaviour of free-edge shallow spherical shells: effect of the geometry, Int. J. Non-linear Mech. 41 (5) (2006) 678-692.

[17] M. Amabili, A comparison of shell theories for large-amplitude vibrations of circular cylindrical shells: Lagrangian approach, J. Sound Vib. 264 (5) (2003) 1091-1125.

[18] M. Amabili, Non-linear vibrations of doubly-curved shallow shells, Int. J. Non-linear Mech. 40 (5) (2005) 683-710.

[19] M. Amabili, Effect of boundary conditions on non-linear vibrations of circular cylindrical panels, ASME J. Appl. Mech. 74 (4) (2007) 645-657.

[20] A. Abe, Y. Kobayashi, G. Yamada, Non-linear vibration characteristics of clamped laminated shallow shells, J. Sound Vib. 234 (2000) 405-426.

[21] I.T. Georgiou, I.B. Schwartz, Slaving the in-plane motions of a nonlinear plate to its flexural motions: an invariant manifold approach, Trans. ASME, J. Appl. Mech. 64 (1997) 175182.

[22] D. Chapelle, K.J. Bathe, The Finite Element Analysis of Shells Fundamentals, Springer-Verlag, Berlin, 2003.

[23] J.H. Ginsberg, Large amplitude forced vibrations of simply supported thin cylindrical shells, J. Appl. Mech. 40 (1973) 471-477.

[24] N. Yamaki, Elastic Stability of Circular Cylindrical Shells, NorthHolland, Amsterdam, 1984.

[25] E.J. Doedel, R. Paffenroth, A.R. Champneys, T.F. Fairgrieve, Y.A. Kuznetsov, B.E. Oldeman, B. Sandstede, X. Wang, AUTO 2000: continuation and bifurcation software for ordinary differential equations, User's guide, Technical Report, Concordia University, 2002.

[26] D. Jiang, Nonlinear modal analysis based on invariant manifolds: application to rotating blade systems, Ph.D. thesis, University of Michigan, 2004.

[27] E. Pesheck, Reduced-order modeling of nonlinear structural systems using nonlinear normal modes and invariant manifolds, Ph.D. thesis, University of Michigan, 2000.

[28] M. Amabili, Theory and experiments for large-amplitude vibrations of circular cylindrical panels with geometric imperfections, J. Sound Vib. 298 (1-2) (2006) 43-72.

[29] A. Steindl, H. Troger, Methods for dimension reduction and their applications in nonlinear dynamics, Int. J. Solids Struct. 38 (2001) 2131-2147. 